\title{
DIVERSITY OF ORCHIDACEAE FROM MURUM DAM, BELAGA, SARAWAK, BORNEO
}

\author{
Ling Chea Yiing ${ }^{1} \&$ Julia Sang ${ }^{2}$
}

\begin{abstract}
Murum Dam in Sarawak is located about $70 \mathrm{~km}$ upstream of Bakun Dam, on the rivers Murum, Danum and Plieran, forming a reservoir over an area of $245 \mathrm{~km}^{2}$. The area consists of mainly lowland to hilly mixed dipterocarp forests, with riparian and alluvial forests along the main rivers and streams, as well as patches of mossy and heath forests. Most of these forests are logged over and some areas have been converted into oil palm plantations. A flora rescue project was carried out to collect selected plant species including orchids from the areas affected by the dam. Most epiphytic orchids were collected from partially submerged trees. Over 2,000 specimens of orchids from 80 genera and c. 276 species were collected from May 2013 to December 2014. The most abundant genus recorded is Bulbophyllum Thouars (c. 44 species). Of these, 37 species are endemic to Borneo, two (Bulbophyllum upupops J.J.Verm., P.O'Byrne \& A.L.Lamb and Thrixspermum lingiae P.O'Byrne \& Gokusing) are newly described and ten species are new records for Sarawak. The collection of species from this work has provided valuable material for the research and conservation of orchids from vulnerable areas around dams.
\end{abstract}

\section{INTRODUCTION}

Approximately 1,100 species of orchids occur in Sarawak (Beaman et al., 2001). According to Beaman et al. (2001), from the inventory work on herbarium specimens collected during the late 19th and early 20th centuries, areas at a lower elevation, from sea level to $300 \mathrm{~m}$, are probably richest in orchid diversity; these areas include lowland and riverine forest. The highest diversity on Mt Kinabalu, however, is found at about 1,500 m elevation. In Sarawak, lowland forest, especially riverine forest, has significantly higher orchid diversity, especially the undisturbed riparian forest where mosses are sometimes present. The trees along the rivers and streams in Murum Dam provide high levels of humidity, creating a suitable microhabitat for orchids to grow (Figs $1 \& 2$ ).

\section{METHODOLOGY}

Plant rescue work was carried out in stages, before, during and after impoundment. An intensive flora assessment survey was carried out in May 2013, before impoundment, in

\footnotetext{
1. Ling Chea Yiing is a Researcher at the Botanical Research Centre, Sarawak Forestry Corporation.

Address: Km 20 Jalan Puncak Borneo, 93250 Kuching, Sarawak, Malaysia.

Email: cyling@sarawakforestry.com

2. Julia Sang is a Researcher at the Botanical Research Centre, Sarawak Forestry Corporation.

Address: as above.

Email: juliasang@sarawakforestry.com
} 


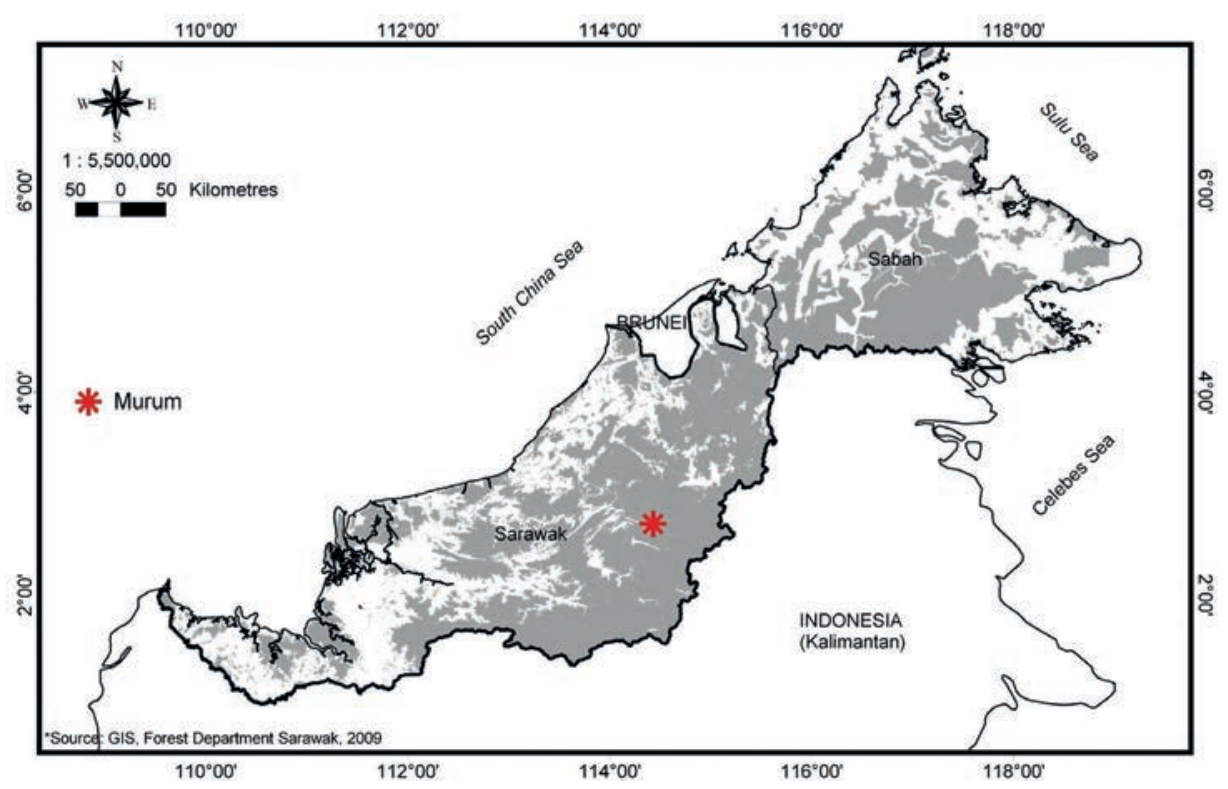

Fig. 1 Map of Sarawak showing the locality of Murum Dam. Map: Ling Chea Yiing.

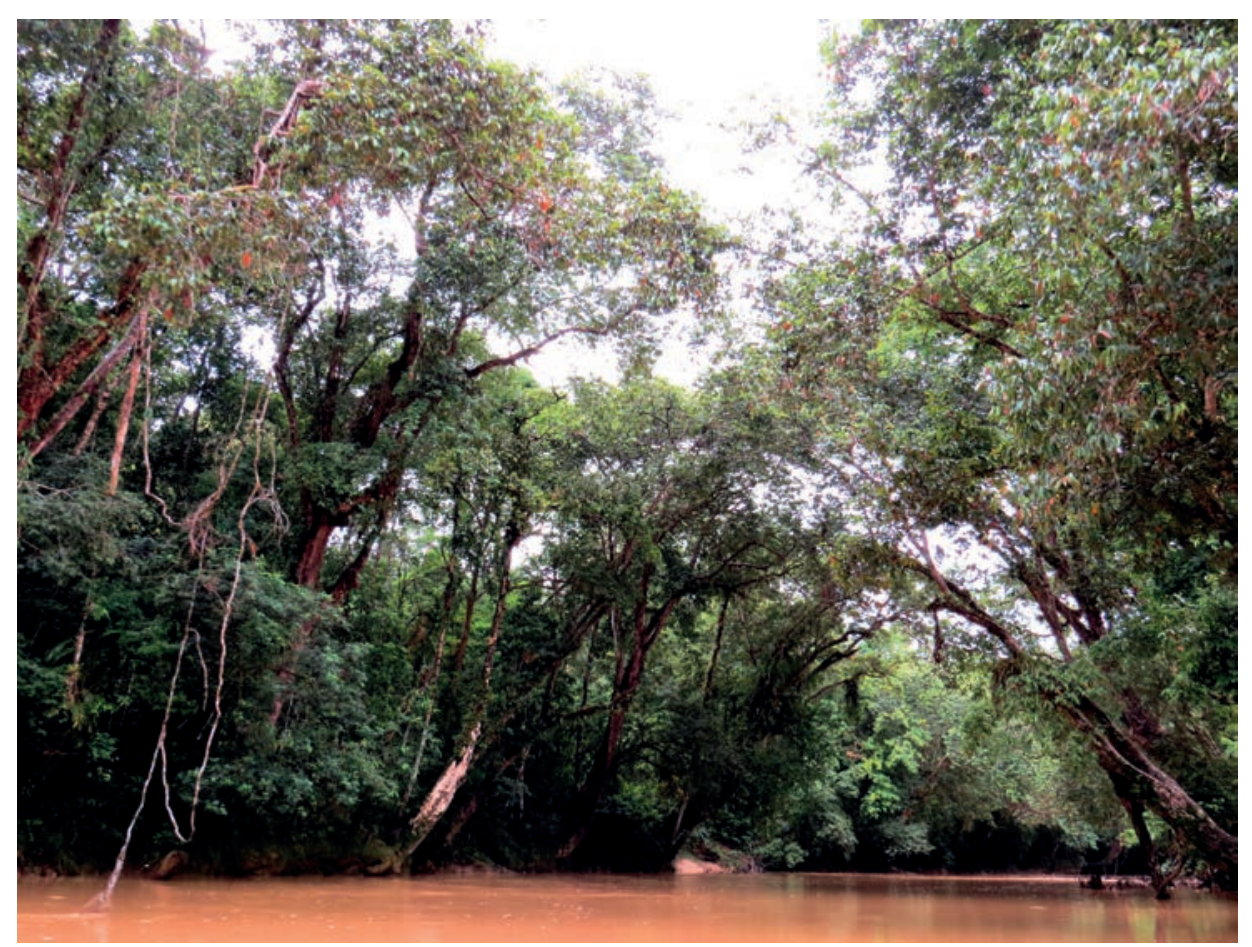

Fig. 2 Ensurai, Dipterocarpus oblongifolius, trees along the river banks at Murum, which provide a suitable microhabitat for epiphytic orchids to grow. Photo: Ling Chea Yiing. 
order to understand the floristic composition and diversity of the flora in the area. From the list of the flora assessed, a list of priority rescue species was drawn up.

Orchidaceae is one of the priority rescue families, as it is one of the Protected Plants listed in the Wildlife Protection Ordinance, 1998 (Laws of Sarawak, 1998). All orchids rescued were tagged and numbered accordingly. Herbarium specimens were made of any flowering orchids and, whenever possible, flowers were preserved in spirit. All specimens are kept in the working herbarium of the Botanical Research Centre (BRC), Kuching, Sarawak. In addition, all flowering specimens were photographed. Identification to species level was carried out by comparing the flowers with the specimens kept in the Kuching Herbarium (SAR), as well as those illustrated in various publications (Ling \& Ong, 2016; O’Byrne, 2016; Vermeulen et al., 2015; Wood, 2014). For species that could not be identified by the authors, more systematic photographs with scale bars were taken so that orchid experts could assist with identification.

During impoundment, flora rescue works were carried out between October 2013 and November 2014 to rescue these selected priority species, with a focus on areas along the three major rivers (Murum, Plieran and Danum) and their tributaries (Fig. 3). During this period, rescue trips were carried out around twice a month, depending on the water level. All plants rescued were planted and grown on at a nursery near the dam site. They were then replanted in the islands that remained after the impoundment. A garden (Murum Flora Conservation Garden) was specifically designed for the planting of all

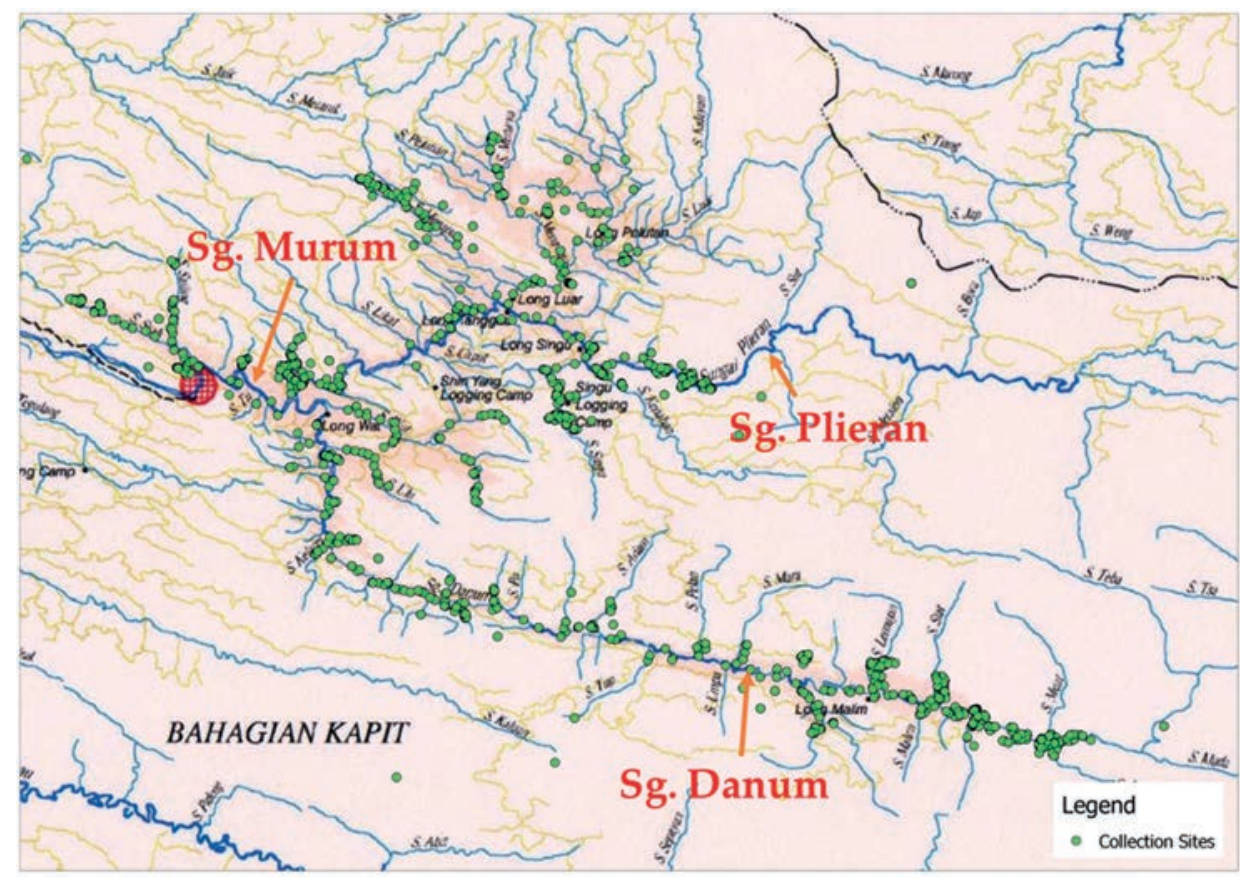

Fig. 3 Map showing the collection sites (in green dots) of the rescue work. Map: Ling Chea Yiing. 
non-tree species, including the orchids, rescued from the submerged forests. In addition, duplicates of non-flowering rescued orchids were grown on in the nursery in the BRC (Botanical Research Centre), Kuching, for monitoring purposes.

\section{FINDINGS AND DISCUSSION}

A total of 2,416 individuals were collected during the rescue works. Of these, c. 276 species from 80 genera were documented. Thirty-seven species are endemic to Borneo (Fig. 4) and ten species are new records for Sarawak (Fig. 5). A checklist of all orchids discovered in Murum is listed in the Appendix. Some of the species have yet to be identified due to a lack of flowering material.
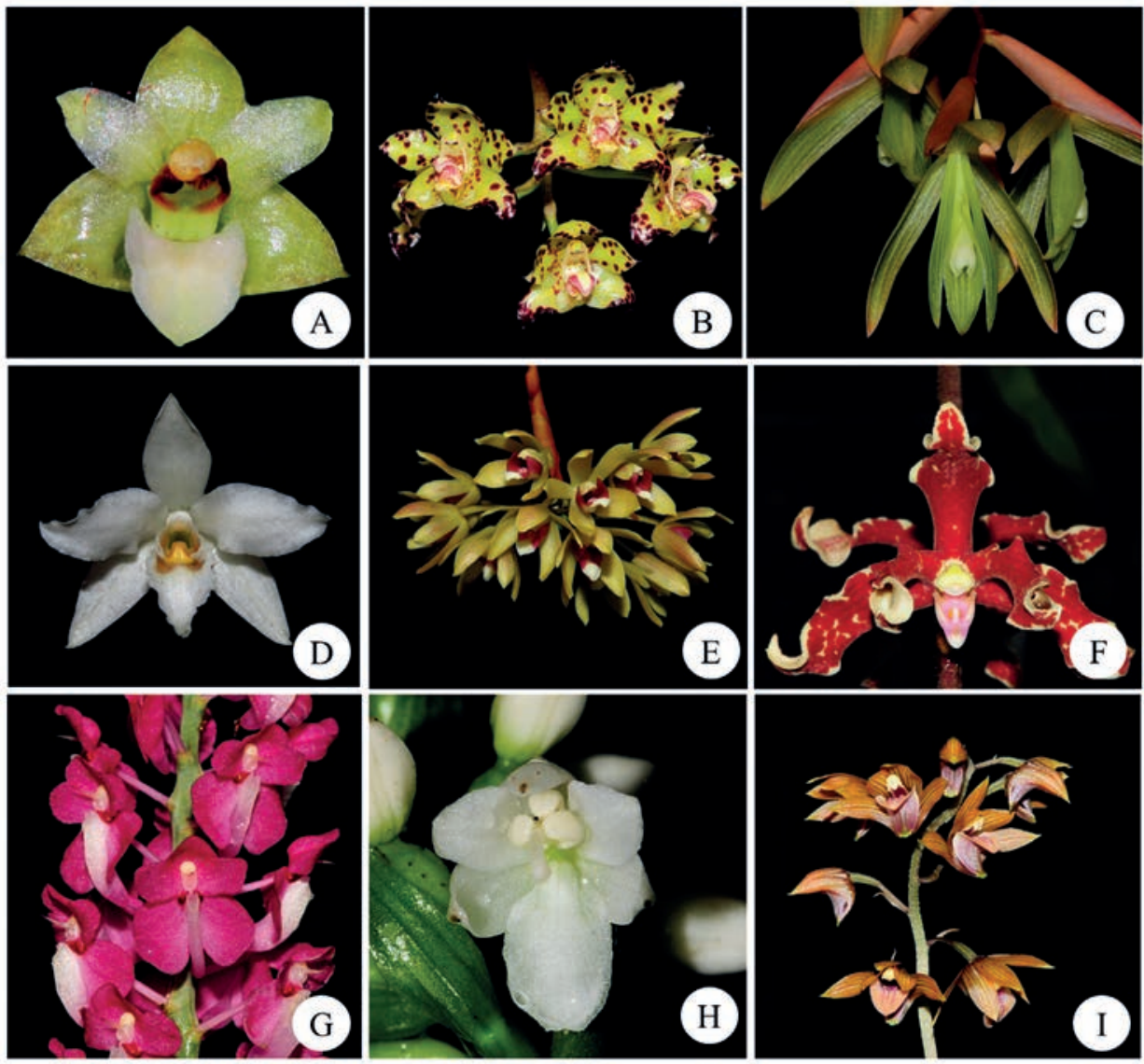

Fig. 4 Some of the orchids that are endemic to Borneo. A Agrostophyllum laterale J.J.Sm.; B Bulbophyllum refractilingue J.J.Sm.; C Coelogyne exalata Ridl.; D Dendrobium tetrachroma Rchb.f.; E Dendrobium speculum J.J.Sm.; F Dimorphorchis lowii (Lindl.) Rolfe; G Dyakia hendersoniana (Rchb.f.) Christenson; H Neuwiedia borneensis de Vogel; and I Tainia scapigera J.J.Sm. Photos: Ling Chea Yiing. 

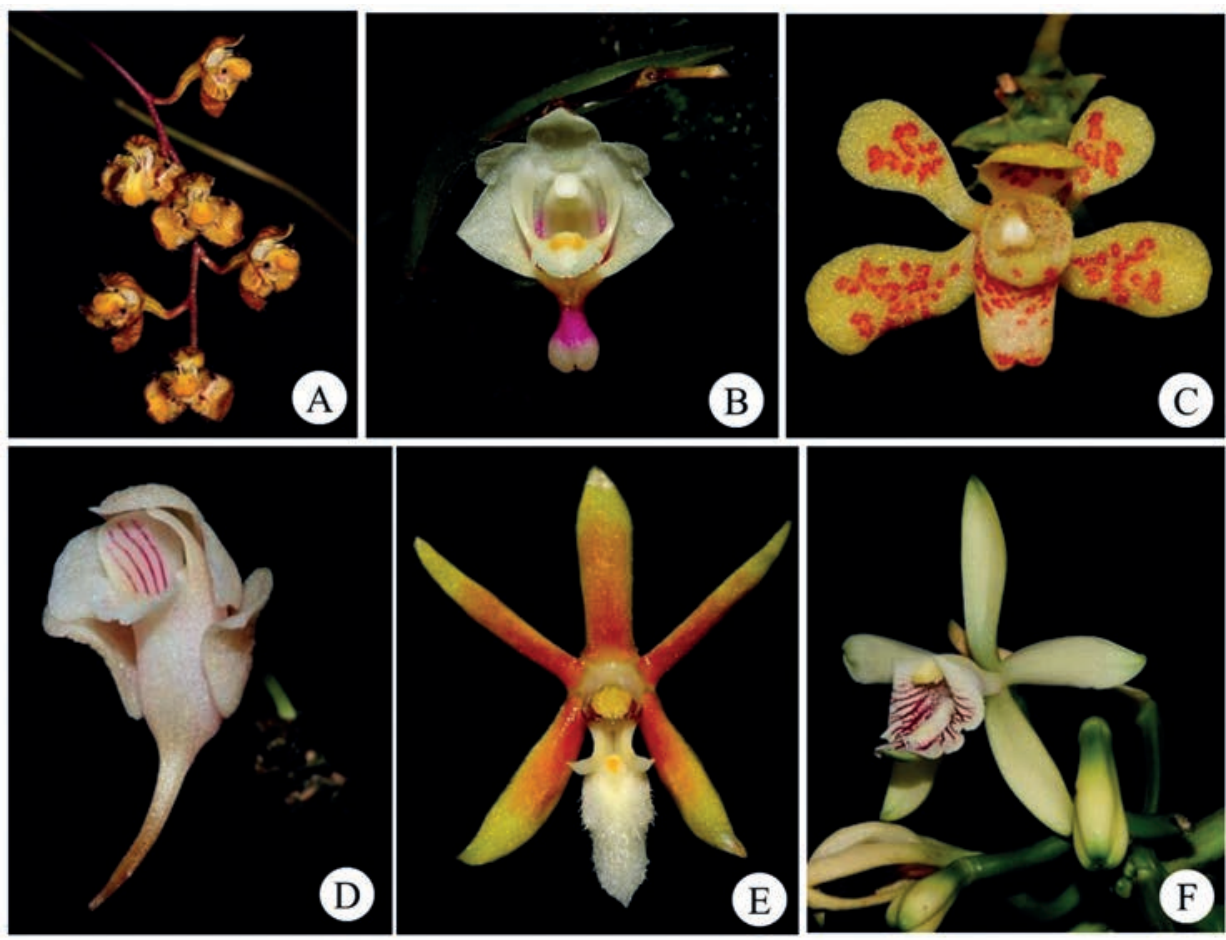

Fig. 5 Some of the orchids that are new records for Sarawak. A Bulbophyllum concavilabium P.O'Byrne \& P.T.Ong; B Dendrobium lancilobum J.J.Wood var. roseocalcar (J.J.Wood \& A.L.Lamb) J.J.Wood; C Thrixspermum pulchrum Carr; D Thrixspermum tenuicalcar Carr; E Trichoglottis persicina P.O'Byrne; F Vanilla sumatrana J.J.Sm. Photos: Ling Chea Yiing.

According to herbarium records at SAR, prior to this project only 66 specimens of orchids were collected from Murum Dam, in 1955 and 2001. This may be due to the remoteness and inaccessibility of the area. As such, this area has not been botanically well explored. In the course of this project, close to 300 species of orchids were discovered. Orchids are not in flower most of the time, and it is almost impossible to identify an orchid without its flower. This project provided an opportunity to monitor the flowering events of the orchids. Monitoring work in the BRC nursery was carried out weekly during the study period. There are no clear patterns to the flowering events of the specimens collected. Some of the orchids flowered within a month after they were collected, most probably due to the stress caused by collection and changes of environment from their original habitat to the nursery. Some collected orchids have to date not produced any flowers or have not survived. The diversity of orchids in Murum Dam can be considered high when compared with other areas such as Mulu National Park (Wood, 1984) and Lanjak Entimau Wildlife Sanctuary (Chai, 2000). During the 1977-1978 expedition to Mulu National Park, Wood reported 174 species of orchids discovered. 
From a single trip or expedition, the chances of obtaining flowering orchids are as low as $5 \%$ or less (through field observations from the past ten years in Sarawak). As such, it will be difficult to determine material to species level unless living plants are collected. Living collections have helped numerous researchers to discover orchids that are new to science (Chan et al., 1994). Monitoring work on flowering events was mainly carried out in the BRC nursery. Monitoring work in the dam site was conducted monthly by the authors for around 12 months before the plants were transplanted in Murum Flora Conservation Garden on a selected permanent island along the Danum River. A total of $30 \%$ of the orchids successfully flowered in BRC and the dam site, which enabled the authors to determine these orchids to species level.

Bulbophyllum is the most abundant genus, followed by Dendrobium and Coelogyne (Table 1). These genera belong to the epiphytic group, which can be found growing abundantly on trees along rivers and streams. During the impoundment, these orchids were easily collected by hand, without the need for tools or to climb trees. Because of this, many more epiphytic species were collected and documented.

During the rescue and monitoring work, many interesting orchids were discovered. These include the two newly described species Bulbophyllum upupops J.J.Verm., P.O'Byrne \& A.L.Lamb (Vermeulen et al., 2015) and Thrixspermum lingiae P.O'Byrne \& Gokusing (O’Byrne, 2016). Bulbophyllum upupops can be found growing on trees along the tributaries of the three main rivers whereas $T$. lingiae is confined to tributaries of the Plieran River, hanging on small branches in riverine forest.

In the first three months of monitoring work in Murum Dam site, the mortality rate of the orchids was about $2 \%$. Most of the more fragile and smaller orchids died, probably due to damage during the transplanting process and the nurserymen's lack of knowledge of how to care for them. Monitoring work on the orchids in the BRC nursery is still ongoing. To date, about 200 plants are still alive in the nursery or have been planted in the BRC orchid garden. Biodiversity rescue is part of the mitigation measures required in the Environmental Impact Assessment (2008) report before any dam construction in Sarawak.

To our knowledge more than 30,000 plants have been rescued, including trees and non-trees. About 52\% of the plants survived and were planted back to the remaining islands at the site of Murum Dam. The plants rescued can never be sufficient to replace

\begin{tabular}{|l|l|}
\hline \multicolumn{1}{|c|}{ Genera } & \multicolumn{1}{c|}{ Total no. of species } \\
\hline Bulbophyllum & 36 \\
\hline Dendrobium & 26 \\
\hline Coelogyne & 16 \\
\hline Dendrochilum & 11 \\
\hline Appendicula & 10 \\
\hline
\end{tabular}

Table 1 The five most abundant genera documented from Murum Dam. 
the biodiversity loss from the dam construction, but through this project, many interesting plants and orchids were discovered. It is a relief that some plants were found growing in other parts of the dam site unaffected by the impoundment. This project has given us an opportunity to study more epiphytic orchids, as many of these orchids are quite impossible to reach without proper climbing gear and experienced field staff.

\section{CONCLUSION}

A high diversity of orchids was discovered thanks to intensive rescue work at Murum Dam. A full species list of orchids discovered at the site has still not been achieved, despite our best efforts to rescue as many plants as possible, because specimens that are either sterile or have no living material could not be identified. A higher diversity of orchids would be expected if all specimens were fertile. Taxonomic studies and monitoring of flowering events are needed in the future for species with no flowering material to date.

Ex situ conservation is part of the outcome from this project. The plants in BRC are a historic representative collection from the dam site and hold important genetic information which may be useful in the future to understand environmental changes and biodiversity loss.

For future rescue work, we would suggest planting the orchids in other areas, such as the area adjacent to the office compound of the Botanical Research Centre. By planting the rescued orchids there, the management and care of the orchids should be more effective. At the same time, the garden could showcase the plants rescued from the dam site and raise local awareness of the wild orchids of Sarawak.

\section{ACKNOWLEDGEMENTS}

The authors would like to thank the organising committee of the 10th Flora Malesiana Symposium for accepting and partially funding the authors to attend the symposium at Royal Botanic Garden Edinburgh. The authors are also grateful to Sarawak Forestry Corporation for allowing the authors to attend the symposium and providing partial funding. Thanks also go to Sarawak Energy Berhad for funding the WiMOR project. The authors would like to acknowledge the assistance of the staff of Sarawak Forestry Corporation and the local communities who were involved in the field collection work. Without their help, this project would not have been possible. The authors are especially grateful to Peter O'Byrne, Ong Poh Teck, Andre Schuiteman and Jaap J. Vermeulen for providing names and assistance in identifying the orchids. 


\section{REFERENCES}

BEAMAN, T.F., WOOD, J.J., BEAMAN, R.S. \& BEAMAN, J.H. (2001). Orchids of Sarawak. Natural History Publications (Borneo), Kota Kinabalu, in association with Royal Botanic Gardens, Kew.

CHAI, P.P.K. (2000). Lanjak Entimau Wildlife Sanctuary, A Check-list of Flora, Fauna, Food and Medicinal Plants. Forest Department, Sarawak \& International Tropical Timber Organization (ITTO).

CHAN, C.L., LAMB, A., SHIM, P.S. \& WOOD, J.J. (1994). Orchids of Borneo. Vol. 1 Introduction and a selection of species. The Sabah Society, Kota Kinabalu, in association with Royal Botanic Gardens, Kew.

ENVIRONMENT IMPACT ASSESSMENT (2008). Proposed Murum Hydroelectric Project, Belaga District, Kapit Division, Sarawak. Chemsain Konsultant Sdn. Bhd., Kuching. Unpublished.

LAWS OF SARAWAK (1998). Wildlife Protection Ordinance 1998 (Chapter 26). Pencetakan Nasional Malaysia Berhad, p. 33.

LING, C.Y. \& ONG, P.T. (2016). Two new records of Vanilla for Sarawak. Malesian Orchid Journal, 18:79-84.

O'BYRNE, P. (2016). Thrixspermum in Borneo: 24 new and old species. Malesian Orchid Journal, 18: 5-74.

VERMEULEN, J., O’BYRNE, P. \& LAMB, A. (2015). Bulbophyllum of Borneo. Natural History Publications (Borneo), Kota Kinabalu.

WOOD, J.J. (1984). A preliminary annotated checklist of the orchids of Gunung Mulu National Park with key to genera. In: JERMY, A.C., Studies on the Flora of Gunung Mulu National Park Sarawak. Forest Department, Kuching, Sarawak, pp. 1-39.

WOOD, J.J. (2014). Dendrobium of Borneo. Natural History Publications (Borneo), Kota Kinabalu, in association with Royal Botanic Gardens, Kew. 


\section{APPENDIX: CHECKLIST OF ORCHIDS DISCOVERED FROM MURUM DAM}

\begin{tabular}{|c|c|c|}
\hline 1 & Acanthophippium javanicum Blume & CMHEP-2700, CMHEP-6061 \\
\hline 2 & Acriopsis liliifolia (J.Koenig) Ormerod & BMHEP-4064, CMHEP-05174 \\
\hline 3 & Acriopsis sp. & CMHEP-2632, АMHEP-2827, BMHEP-2249 \\
\hline 4 & Aerides odorata Lour. & $\begin{array}{l}\text { CMHEP-0237, CMHEP-2808, CMHEP-2845, } \\
\text { CMHEP-1351, BMHEP-3999, CMHEP-03618, } \\
\text { BMHEP-03674, CMHEP-5996, CMHEP-3302, } \\
\text { CMHEP-6543, CMHEP-0236, AMHEP-1092 }\end{array}$ \\
\hline 5 & Agrostophyllum cyathiforme J.J.Sm. & AMHEP-894, CMHEP-5935 \\
\hline 6 & Agrostophyllum laterale J.J.Sm. & $\begin{array}{l}\text { BMHEP-2397, BMHEP-1615, BMHEP-3920 } \\
\text { (endemic to Borneo) }\end{array}$ \\
\hline 7 & Agrostophyllum saccatum Ridl. & BMHEP-3972 \\
\hline 8 & $\begin{array}{l}\text { Agrostophyllum sumatranum Schltr. \& } \\
\text { J.J.Sm. }\end{array}$ & BMHEP-3803 \\
\hline 9 & Agrostophyllum stipulatum Schltr. & CMHEP-2210, ВMHEP-3982 \\
\hline 10 & Agrostophyllum spp. & $\begin{array}{l}\text { BMHEP-1402, BMHEP-2152, BMHEP-2513, } \\
\text { CMHEP-1361 }\end{array}$ \\
\hline 11 & Anoectochilus sp. & CMHEP-03629 \\
\hline 12 & Aphyllorchis montana Rchb.f. & CMHEP-0051, CMHEP-0161 \\
\hline 13 & Apostasia wallichii R.Br. & CMHEP-0536, BMHEP-3863 \\
\hline 14 & Appendicula anceps Blume & AMHEP-870, BMHEP-2415 \\
\hline 15 & Appendicula cornuta Blume & CMHEP-0064 \\
\hline 16 & Appendicula cristata Blume & AMHEP-1071, CMHEP-5910, CMHEP-06217 \\
\hline 17 & $\begin{array}{l}\text { Appendicula longirostrata Ames \& } \\
\text { C.Schweinf. }\end{array}$ & $\begin{array}{l}\text { CMHEP-05088, CMHEP-5905, CMHEP-5956 } \\
\text { (endemic to Borneo) }\end{array}$ \\
\hline 18 & Appendicula pendula Blume & AMHEP-0204, CMHEP-6101 \\
\hline 19 & Appendicula recondita J.J.Sm. & CMHEP-0159, BMHEP-3840 (endemic to Borneo) \\
\hline 20 & Appendicula reflexa Blume & CMHEP-2633, CMHEP-2942 \\
\hline 21 & Appendicula torta Blume & AMHEP-0440, AMHEP-3198 \\
\hline 22 & Appendicula spp. & BMHEP-0296, СMHEP-2212, CMHEP-1335 \\
\hline 23 & Arundina graminifolia Hochr. & CMHEP-1441, BMHEP-1691 \\
\hline 24 & Ascochilus emarginatus (Blume) Schuit. & CMHEP-1437 \\
\hline 25 & Bromheadia finlaysoniana (Lindl.) Miq. & AMHEP-1292, BMHEP-3862-1 \\
\hline 26 & Bromheadia sp. & BMHEP-1607 \\
\hline 27 & $\begin{array}{l}\text { Bryobium hyacinthoides (Blume) Y.P.Ng } \\
\text { \& P.J.Cribb }\end{array}$ & $\begin{array}{l}\text { AMHEP-0418, AMHEP-0463, AMHEP-1284, } \\
\text { BMHEP-4010, CMHEP-6083 }\end{array}$ \\
\hline 28 & Bryobium sp. & BMHEP-1401, ВМHЕР-1528 \\
\hline 29 & Bulbophyllum acuminatum Ridl. & AMHEP-890, CMHEP-2259 \\
\hline
\end{tabular}




\begin{tabular}{|c|c|c|}
\hline 30 & Bulbophyllum antenniferum Rchb.f. & BMHEP-2966 \\
\hline 31 & Bulbophyllum apodum Hook.f. & $\begin{array}{l}\text { CMHEP-0980, CMHEP-0233, CMHEP-1331, } \\
\text { CMHEP-05171, CMHEP-6516, CMHEP-05156, } \\
\text { AMHEP-2825, BMHEP-2862, CMHEP-1343 }\end{array}$ \\
\hline 32 & Bulbophyllum armeniacum J.J.Sm. & AMHEP-1175, AMHEP-1104 \\
\hline 33 & $\begin{array}{l}\text { Bulbophyllum artvogelii J.J.Verm., } \\
\text { P.O'Byrne \& A.L.Lamb }\end{array}$ & BMHEP-2850, BMHEP-3814, СMHEP-06216 \\
\hline 34 & Bulbophyllum biseriale Carr & BMHEP-3893, CMHEP-5933 \\
\hline 35 & $\begin{array}{l}\text { Bulbophyllum caudatisepalum Ames \& } \\
\text { C.Schweinf. }\end{array}$ & CMHEP-0134 \\
\hline 36 & Bulbophyllum cf. caecilii J.J.Sm. & CMHEP-1332 \\
\hline 37 & Bulbophyllum cf. mahakamense J.J.Sm. & AMHEP-451, AMHEP-1142, BMHEP-3856 \\
\hline 38 & Bulbophyllum cf. osyricera Schltr. & BMHEP-4036 \\
\hline 39 & Bulbophyllum cf. virescens J.J.Sm. & CMHEP-2790 \\
\hline 40 & $\begin{array}{l}\text { Bulbophyllum concavilabium P.O’Byrne } \\
\text { \& P.T.Ong }\end{array}$ & AMHEP-839 (new record) \\
\hline 41 & Bulbophyllum coniferum Ridl. & BMHEP-3851, BMHEP-4009 \\
\hline 42 & Bulbophyllum ecornutum J.J.Sm. & BMHEP-2247 \\
\hline 43 & Bulbophyllum flavescens Lindl. & AMHEP-852, BMHEP-4049 \\
\hline 44 & Bulbophyllum hirtulum Ridl. & CMHEP-201 \\
\hline 45 & Bulbophyllum lasianthum Lindl. & AMHEP-1114, CMHEP-6571 \\
\hline 46 & Bulbophyllum laxiflorum Lindl. & $\begin{array}{l}\text { AMHEP-1106, BMHEP-2954, BMHEP-2592, } \\
\text { BMHEP-3848 }\end{array}$ \\
\hline 47 & Bulbophyllum lobbii Lindl. & BMHEP-4068, BMHEP-4101 \\
\hline 48 & Bulbophyllum macrochilum Rolfe & AMHEP-1285 \\
\hline 49 & Bulbophyllum membranifolium Hook.f. & CMHEP-4292 \\
\hline 50 & Bulbophyllum odoratum Lindl. & BMHEP-2388, CMHEP-2646 \\
\hline 51 & Bulbophyllum otochilum J.J.Verm. & CMHEP-1830 (endemic to Borneo) \\
\hline 52 & Bulbophyllum penduliscapum J.J.Sm. & CMHEP-1347, СMHEP-6555, СMHЕР-6572 \\
\hline 53 & Bulbophyllum puguahaanense Ames & AMHEP-1087 (new record) \\
\hline 54 & $\begin{array}{l}\text { Bulbophyllum purpurascens Teijsm. \& } \\
\text { Binn. }\end{array}$ & CMHEP-2749 \\
\hline 55 & Bulbophyllum putidum J.J.Sm. & CMHEP-5940 \\
\hline 56 & Bulbophyllum refractilingue J.J.Sm. & $\begin{array}{l}\text { CMHEP-2264, BMHEP-1367, BMHEP-4041, } \\
\text { BMHEP-04741, BMHEP-1912 (endemic to Borneo) }\end{array}$ \\
\hline 57 & Bulbophyllum simii J.J.Verm. \& A.L.Lamb & AMHEP-1128 (endemic to Borneo) \\
\hline 58 & Bulbophyllum sulcatum Lindl. & CMHEP-05127-1 \\
\hline 59 & Bulbophyllum tortuosum Lindl. & AMHEP-1107 \\
\hline
\end{tabular}




\begin{tabular}{|c|c|c|}
\hline 60 & Bulbophyllum trifolium Ridl. & BMHEP-2442 \\
\hline 61 & $\begin{array}{l}\text { Bulbophyllum upupops J.J.Verm., } \\
\text { P.O’Byrne \& A.L.Lamb }\end{array}$ & BMHEP-3878, CMHEP-4313 (endemic to Borneo) \\
\hline 62 & Bulbophyllum vaginatum Rchb.f. & CMHEP-2813, AMHEP-2836 \\
\hline 63 & Bulbophyllum vermiculare Hook.f. & AMHEP-1108 \\
\hline 64 & Bulbophyllum sp. & AMHEP-1016 \\
\hline 65 & Bulbophyllum spp. & $\begin{array}{l}\text { AMHEP-885, CMHEP-0232, CMHEP-0234, } \\
\text { CMHEP-0259, BMHEP-0298, BMHEP-1604, } \\
\text { BMHEP-1606, BMHEP-1710, BMHEP-1722, } \\
\text { CMHEP-2674, CMHEP-2816, AMHEP-2826, } \\
\text { AMHEP-2832, AMHEP-2839, BMHEP-2860, } \\
\text { CMHEP-1313, CMHEP-1322, CMHEP-1341, } \\
\text { CMHEP-1355, BMHEP-1368, CMHEP-1397, } \\
\text { CMHEP-0903, CMHEP-05004, CMHEP-05021, } \\
\text { CMHEP-05025, CMHEP-05077, CMHEP-05078, } \\
\text { CMHEP-05105, CMHEP-05175, CMHEP-05188, } \\
\text { AMHEP-1062, BMHEP-2418, MHEP-4146 }\end{array}$ \\
\hline 66 & Calanthe pulchra Lindl. & $\begin{array}{l}\text { BMHEP-1579, ВMHEP-1656, ВMHEP- } 1675 \text {, } \\
\text { BMHEP-4066, ВMHEP-04539, ВМHEP-06400 }\end{array}$ \\
\hline 67 & Calanthe vestita Lindl. & BMHEP-04742 \\
\hline 68 & Calanthe sp. & BMHEP-1420, BMHEP-04748 \\
\hline 69 & $\begin{array}{l}\text { Callostylis pulchella (Lindl.) S.C.Chen \& } \\
\text { Z.H.Tsi }\end{array}$ & $\begin{array}{l}\text { AMHEP-823, AMHEP-0475, BMHEP-0294, } \\
\text { BMHEP-2119, BMHEP-3986, CMHEP-05013, } \\
\text { CMHEP-05173 }\end{array}$ \\
\hline 70 & $\begin{array}{l}\text { Campanulorchis leiophylla (Lindl.) Y.P.Ng } \\
\& \text { P.J.Cribb }\end{array}$ & CMHEP-1356, CMHEP-05050 \\
\hline 71 & $\begin{array}{l}\text { Campanulorchis pseudoleiophylla } \\
\text { (J.J.Wood) J.J.Wood }\end{array}$ & AMHEP-1001 \\
\hline 72 & $\begin{array}{l}\text { Ceratostylis cf. octomerioides J.J.Woods } \\
\text { \& A.L.Lamb }\end{array}$ & AMHEP-2364 \\
\hline 73 & Ceratostylis pendula Hook.f. & BMHEP-4056 \\
\hline 74 & Ceratostylis sp.1 & CMHEP-1348 \\
\hline 75 & Chelonistele ingloria (J.J.Sm.) Carr & CMHEP-0249 (endemic to Borneo) \\
\hline 76 & Chelonistele sulphurea Pfitzer & CMHEP-2819, BMHEP-1372, BMHEP-4038 \\
\hline 77 & Chelonistele sp.1 & CMHEP-2731 \\
\hline 78 & Claderia viridiflora Hook.f. & CMHEP-0505, ВMHEP-04959 \\
\hline 79 & Cleisostoma suaveolens Blume & $\begin{array}{l}\text { CMHEP-2811, BMHEP-04792, BMHEP-6903, } \\
\text { BMHEP-3947 }\end{array}$ \\
\hline 80 & Cleisostoma spp. & BMHEP-3881, CMHEP-03638 \\
\hline 81 & Coelogyne asperata Lindl. & BMHEP-2401, CMHEP-2817, ВMHEP-2581 \\
\hline 82 & Coelogyne cf. mayeriana Rchb.f. & CMHEP-5946 \\
\hline 83 & Coelogyne endertii J.J.Sm. & CMHEP-659 (endemic to Borneo) \\
\hline
\end{tabular}




\begin{tabular}{|c|c|c|}
\hline 84 & Coelogyne exalata Ridl. & CMHEP-271 (endemic to Borneo) \\
\hline 85 & Coelogyne foerstermanni Rchb.f. & CMHEP-2677, CMHEP-1379, CMHEP-06340 \\
\hline 86 & Coelogyne gibbifera J.J.Sm. & CMHEP-03614 \\
\hline 87 & Coelogyne incrassata Lindl. & AMHEP-1051, CMHEP-6077 \\
\hline 88 & Coelogyne mayeriana Rchb.f. & $\begin{array}{l}\text { AMHEP-1100, BMHEP-1506, CMHEP-05042, } \\
\text { CMHEP-06300 }\end{array}$ \\
\hline 89 & Coelogyne pandurata Lindl. & $\begin{array}{l}\text { AMHEP-0469, CMHEP-0265, CMHEP-2678, } \\
\text { CMHEP-1304, CMHEP-5947 }\end{array}$ \\
\hline 90 & Coelogyne pholidotoides J.J.Sm. & AMHEP-1116 (endemic to Borneo) \\
\hline 91 & Coelogyne pulverula Teijsm. \& Binn. & $\begin{array}{l}\text { AMHEP-0425, BMHEP-04510, BMHEP-04733, } \\
\text { BMHEP-04920, BMHEP-05784, CMHEP-6553, } \\
\text { BMHEP-2399 }\end{array}$ \\
\hline 92 & Coelogyne rochusseni de Vriese & $\begin{array}{l}\text { BMHEP-1642, BMHEP-2972, CMHEP-2814, } \\
\text { BMHEP-3811, BMHEP-3910, CMHEP-05148, } \\
\text { CMHEP-5922, BMHEP-05740 }\end{array}$ \\
\hline 93 & Coelogyne sanderiana Rchb.f & $\begin{array}{l}\text { AMHEP-1014, AMHEP-0205, CMHEP-2771, } \\
\text { CMHEP-1400 }\end{array}$ \\
\hline 94 & Coelogyne septemcostata J.J.Sm. & $\begin{array}{l}\text { CMHEP-2745, BMHEP-2154, BMHEP-2855, } \\
\text { CMHEP-03601, CMHEP-03701, CMHEP-5963 }\end{array}$ \\
\hline 95 & Coelogyne swaniana Rolfe & $\begin{array}{l}\text { BMHEP-2449, AMHEP-2542, BMHEP-03656, } \\
\text { BMHEP-05785, BMHEP-3343, CMHEP-6545, } \\
\text { AMHEP-0211 }\end{array}$ \\
\hline 96 & Coelogyne testacea Lindl. & CMHEP-5904 \\
\hline 97 & Coelogyne spp. & $\begin{array}{l}\text { CMHEP-0242, CMHEP-0262, BMHEP-1728, } \\
\text { BMHEP-2863, BMHEP-2864, BMHEP-2893, } \\
\text { BMHEP-2569, CMHEP-2265, CMHEP-1333, } \\
\text { CMHEP-1396, AMHEP-1288, CMHEP-0611, } \\
\text { CMHEP-05141, CMHEP-06203, CMHEP-06358, } \\
\text { CMHEP-05012 }\end{array}$ \\
\hline 98 & Collabium cf. simplex Rchb.f. & $\begin{array}{l}\text { CMHEP-6601, (live plant planted in garden at dam } \\
\text { site) }\end{array}$ \\
\hline 99 & Corymborkis veratrifolia (Reinw.) Blume & AMHEP-1275, CMHEP-2904 \\
\hline 100 & $\begin{array}{l}\text { Crepidium damusicum (J.J.Sm.) Marg. \& } \\
\text { Szlach. }\end{array}$ & AMHEP-1133, CMHEP-6006 (endemic to Borneo) \\
\hline 101 & Crepidium micranthum (Hook.f.) Szlach. & BMHEP-04967 \\
\hline 102 & $\begin{array}{l}\text { Cymbidium bicolor Lindl. subsp. } \\
\text { pubescens (Lindl.) Du Puy \& P.J.Cribb }\end{array}$ & BMHEP-4022, BMHEP-04515 \\
\hline 103 & Cymbidium finlaysonianum Lindl. & CMHEP-6557 \\
\hline 104 & Cymbidium spp. & $\begin{array}{l}\text { AMHEP-0213, CMHEP-0229, CMHEP-2666, } \\
\text { CMHEP-2810, CMHEP-2202, BMHEP-3895 }\end{array}$ \\
\hline 105 & Cystorchis javanica (Blume) Blume & BMHEP-4067 \\
\hline 106 & Cystorchis variegata Blume & CMHEP-03626 \\
\hline
\end{tabular}




\begin{tabular}{|c|c|c|}
\hline 107 & Dendrobium acerosum Lindl. & AMHEP-806, BMHEP-04490 \\
\hline 108 & Dendrobium bancanum J.J.Sm. & CMHEP-2846, СMHEP-2261, CMHEP-2268 \\
\hline 109 & Dendrobium bicostatum J.J.Sm. & CMHEP-2847 \\
\hline 110 & $\begin{array}{l}\text { Dendrobium connatum var. connatum } \\
\text { (Blume) Lindl. }\end{array}$ & $\begin{array}{l}\text { AMHEP-2351, AMHEP-0576, CMHEP-1820, } \\
\text { BMHEP-2246, CMHEP-1375, BMHEP-3953, } \\
\text { CMHEP-03641, BMHEP-04526, BMHEP-05703, } \\
\text { CMHEP-6576 }\end{array}$ \\
\hline 111 & Dendrobium endertii J.J.Sm. & AMHEP-2114 (endemic to Borneo) \\
\hline 112 & Dendrobium geminatum (Blume) Lindl. & CMHEP-2176, СMHEР-5926, ВМНЕР-6911 \\
\hline 113 & $\begin{array}{l}\text { Dendrobium hendersonii A.D.Hawkes \& } \\
\text { A.H.Heller }\end{array}$ & BMHEP-2394 \\
\hline 114 & Dendrobium heterocarpum Wall. ex Lindl. & BMHEP-2385, BMHEP-04511, BMHEP-04943 \\
\hline 115 & Dendrobium hosei Ridl. & $\begin{array}{l}\text { AMHEP-831, CMHEP-0496, CMHEP-0528, } \\
\text { CMHEP-0244, CMHEP-1454, BMHEP-1556, } \\
\text { BMHEP-1649, CMHEP-2769, CMHEP-1354, } \\
\text { CMHEP-5953, BMHEP-05819 }\end{array}$ \\
\hline 116 & $\begin{array}{l}\text { Dendrobium lancilobum var. roseocalcar } \\
\text { (J.J.Wood \& A.L.Lamb) J.J.Wood }\end{array}$ & CMHEP-0079 (endemic to Borneo; new record) \\
\hline 117 & Dendrobium lobbii Teijsm. \& Binn. & CMHEP-0532, CMHEP-6076 \\
\hline 118 & Dendrobium luxurians J.J.Sm. & CMHEP-6026 \\
\hline 119 & Dendrobium microglaphys Rchb.f. & $\begin{array}{l}\text { BMHEP-1723, BMHEP-3018, CMHEP-1398, } \\
\text { AMHEP-1068 }\end{array}$ \\
\hline 120 & Dendrobium planibulbe Lindl. & BMHEP-04513 \\
\hline 121 & $\begin{array}{l}\text { Dendrobium pleasancium P.O'Byrne \& } \\
\text { J.J.Verm. }\end{array}$ & CMHEP-05187 (endemic to Borneo) \\
\hline 122 & Dendrobium rosellum Ridl. & $\begin{array}{l}\text { AMHEP-807, CMHEP-0231, CMHEP-0243, } \\
\text { BMHEP-1683, CMHEP-1363, BMHEP-4104 }\end{array}$ \\
\hline 123 & $\begin{array}{l}\text { Dendrobium secundum (Blume) Lindl. } \\
\text { ex Wall. }\end{array}$ & BMHEP-3991 \\
\hline 124 & Dendrobium setifolium Ridl. & BMHEP-2393 \\
\hline 125 & Dendrobium spathipetalum J.J.Sm. & $\begin{array}{l}\text { CMHEP-2476, CMHEP-0495, BMHEP- } 6917 \\
\text { (endemic to Borneo) }\end{array}$ \\
\hline 126 & Dendrobium speculum J.J.Sm. & $\begin{array}{l}\text { AMHEP-834, AMHEP-893, BMHEP-2412, } \\
\text { BMHEP-1720, BMHEP-3883, BMHEP-3979 } \\
\text { (endemic to Borneo) }\end{array}$ \\
\hline 127 & Dendrobium stuposum Lindl. & ВМНЕР-2391, ВМНЕР-3901, ВМНЕР-3937 \\
\hline 128 & Dendrobium tetrachromum Rchb.f. & $\begin{array}{l}\text { CMHEP-2964, BMHEP-2965, BMHEP-3815 } \\
\text { (endemic to Borneo) }\end{array}$ \\
\hline 129 & $\begin{array}{l}\text { Dendrobium treacherianum Rchb.f. ex } \\
\text { Hook.f. }\end{array}$ & $\begin{array}{l}\text { BMHEP-1721, CMHEP-2262, CMHEP-1318, } \\
\text { BMHEP-1366, CMHEP-1374, BMHEP-2150, } \\
\text { BMHEP-1935 }\end{array}$ \\
\hline 130 & Dendrobium xantholeucum Rchb.f. & CMHEP-2206 \\
\hline
\end{tabular}




\begin{tabular}{|c|c|c|}
\hline 131 & Dendrobium spp. & $\begin{array}{l}\text { CMHEP-1450, CMHEP-2649, CMHEP-2746, } \\
\text { CMHEP-2752, CMHEP-2178, AMHEP-2831, } \\
\text { BMHEP-2576, CMHEP-2209, BMHEP-2245, } \\
\text { CMHEP-1312, CMHEP-1357, BMHEP-3850, } \\
\text { CMHEP-5016, CMHEP-5051, CMHEP-5172, } \\
\text { CMHEP-6366, BMHEP-4633, CMHEP-6298 }\end{array}$ \\
\hline 132 & Dendrochilum anomalum Carr & CMHEP-1337, BMHEP-2327 (endemic to Borneo) \\
\hline 133 & $\begin{array}{l}\text { Dendrochilum cruciforme var. longicuspe } \\
\text { J.J.Wood }\end{array}$ & AMHEP-851, CMHEP-2461 (endemic to Borneo) \\
\hline 134 & Dendrochilum integrilabium Carr & CMHEP-6091, CMHEP-06351 (endemic to Borneo) \\
\hline 135 & Dendrochilum kingii (Hook.f.) J.J.Sm. & $\begin{array}{l}\text { AMHEP-869, AMHEP-1111, AMHEP-1191, } \\
\text { CMHEP-0501, CMHEP-0270, BMHEP-1370, } \\
\text { BMHEP-3810, BMHEP-3921, BMHEP-04451, } \\
\text { BMHEP-04789, BMHEP-04919 }\end{array}$ \\
\hline 136 & Dendrochilum longifolium Rchb.f. & AMHEP-1110 \\
\hline 137 & Dendrochilum longipes J.J.Sm. & CMHEP-2775 (endemic to Borneo) \\
\hline 138 & Dendrochilum oxylobum Schltr. & BMHEP-4115 (endemic to Borneo) \\
\hline 139 & Dendrochilum pallidiflavens Blume & $\begin{array}{l}\text { AMHEP-853, AMHEP-1182, CMHEP-0241, } \\
\text { CMHEP-0272, CMHEP-2770, CMHEP-1350, } \\
\text { CMHEP-1385, CMHEP-03645, CMHEP-06354, } \\
\text { CMHEP-5915, AMHEP-1006, CMHEP-6070 }\end{array}$ \\
\hline 140 & Dendrochilum pubescens L.O.Williams & AMHEP-0407 (endemic to Borneo) \\
\hline 141 & Dendrochilum rufum (Rolfe) J.J.Sm. & $\begin{array}{l}\text { CMHEP-0526, CMHEP-1464, CMHEP-1492, } \\
\text { BMHEP-1551, CMHEP-6049 (endemic to Borneo) }\end{array}$ \\
\hline 142 & Dendrochilum spp. & $\begin{array}{l}\text { AMHEP-0207, CMHEP-0283, BMHEP-1603, } \\
\text { CMHEP-1302, CMHEP-2802, CMHEP-1317, } \\
\text { CMHEP-05047 }\end{array}$ \\
\hline 143 & Dienia ophrydis (J.Koenig) Seidenf. & AMHEP-1058 \\
\hline 144 & Dilochia rigida (Ridl.) J.J.Wood & CMHEP-4214 (endemic to Borneo) \\
\hline 145 & Dilochia wallichii Lindl. & CMHEP-0055, СMHEP-2809, BMHEP-04795 \\
\hline 146 & Dilochia sp. & CMHEP-1359, СMHEP-05019 \\
\hline 147 & Dimorphorchis lowii (Lindl.) Rolfe & $\begin{array}{l}\text { CMHEP-2823, BMHEP-05719, CMHEP-6574 } \\
\text { (endemic to Borneo) }\end{array}$ \\
\hline 148 & Dipodium purpureum J.J.Sm. & CMHEP-0252, CMHEP-2735, ВMHEP-2416 \\
\hline 149 & Dipodium sp.1 & BMHEP-2223 \\
\hline 150 & $\begin{array}{l}\text { Dyakia hendersoniana (Rchb.f.) } \\
\text { Christenson }\end{array}$ & $\begin{array}{l}\text { AMHEP-2842, BMHEP-4046, CMHEP-03624 } \\
\text { (endemic to Borneo) }\end{array}$ \\
\hline 151 & $\begin{array}{l}\text { Entomophobia kinabaluensis (Ames) de } \\
\text { Vogel }\end{array}$ & $\begin{array}{l}\text { CMHEP-2460, CMHEP-0522, CMHEP-0246, } \\
\text { CMHEP-2774, AMHEP-2837, CMHEP-2260, } \\
\text { CMHEP-1387, CMHEP-6028 }\end{array}$ \\
\hline 152 & Eria crassipes Ridl. & CMHEP-1316, СMHEP-03623 \\
\hline 153 & Eria javanica (Sw.) Blume & CMHEP-6531 \\
\hline 154 & Eria neglecta Ridl. & CMHEP-2472 \\
\hline
\end{tabular}




\begin{tabular}{|c|c|c|}
\hline 155 & Eria nutans Lindl. & CMHEP-2175, CMHEP-6562 \\
\hline 156 & Eria robusta (Blume) Lindl. & $\begin{array}{l}\text { CMHEP-0523, BMHEP-1719, CMHEP-2779, } \\
\text { AMHEP-2829, CMHEP-1384, BMHEP-3861, } \\
\text { BMHEP-4118, BMHEP-04518, CMHEP-05108, } \\
\text { CMHEP-5921, BMHEP-2389 }\end{array}$ \\
\hline 157 & Eria spp. & $\begin{array}{l}\text { CMHEP-2457, CMHEP-0538, AMHEP-1283, } \\
\text { CMHEP-0981, CMHEP-0247, CMHEP-0253, } \\
\text { CMHEP-0255, CMHEP-0256, CMHEP-0261, } \\
\text { BMHEP-0297, BMHEP-1617, BMHEP-1618, } \\
\text { BMHEP-1648, CMHEP-2671, CMHEP-2675, } \\
\text { CMHEP-2676, CMHEP-2806, CMHEP-2807, } \\
\text { CMHEP-2824, AMHEP-2835, CMHEP-2205, } \\
\text { CMHEP-2211, BMHEP-2250, CMHEP-2254, } \\
\text { CMHEP-2255, CMHEP-1326, CMHEP-1345, } \\
\text { BMHEP-1365, BMHEP-4008, CMHEP-05010, } \\
\text { CMHEP-05028, CMHEP-05071, CMHEP-05126, } \\
\text { CMHEP-05185, CMHEP-05189, CMHEP-2181, } \\
\text { CMHEP-3308, BMHEP-04496 }\end{array}$ \\
\hline 158 & Geesinkorchis sp. & $\begin{array}{l}\text { CMHEP-0245, CMHEP-1496, CMHEP-1632, } \\
\text { AMHEP-2838, CMHEP-2267, CMHEP-1315, } \\
\text { BMHEP-4037, CMHEP-5930, AMHEP-815, } \\
\text { BMHEP-6908 }\end{array}$ \\
\hline 159 & Grammatophyllum speciosum Blume & $\begin{array}{l}\text { CMHEP-0524, CMHEP-0021, CMHEP-1349, } \\
\text { CMHEP-3193, CMHEP-05075 }\end{array}$ \\
\hline 160 & Hylophila cheangii Holttum & CMHEP-5984, CMHEP-6051 \\
\hline 161 & Lecanorchis multiflora J.J.Sm. & CMHEP-5972 \\
\hline 162 & Liparis barbata Lindl. & $\begin{array}{l}\text { AMHEP-1041, AMHEP-0436, CMHEP-2736, } \\
\text { BMHEP-3028, BMHEP-3911, CMHEP-5964 }\end{array}$ \\
\hline 163 & Liparis cespitosa (Lam.) Lindl. & CMHEP-2458 \\
\hline 164 & Liparis elegans Lindl. & BMHEP-2881, BMHEP-3854 \\
\hline 165 & Liparis grandiflora Ridl. & AMHEP-1272, BMHEP-3806 (endemic to Borneo) \\
\hline 166 & Liparis lacerata Ridl. & $\begin{array}{l}\text { AMHEP-858, AMHEP-0419, CMHEP-0116, } \\
\text { BMHEP-3994, CMHEP-05011 }\end{array}$ \\
\hline 167 & Liparis latifolia Lindl. & BMHEP-04941 \\
\hline 168 & Liparis rheedei Lindl. & BMHEP-1580, СMHЕР-6541, ВМНЕР-04908 \\
\hline 169 & Liparis spp. & $\begin{array}{l}\text { BMHEP-3027, BMHEP-0684, BMHEP-04502, } \\
\text { BMHEP-04540, BMHEP-05730 }\end{array}$ \\
\hline 170 & Luisia antennifera Blume & CMHEP-1376, CMHEP-05079, CMHEP-5931 \\
\hline 171 & Macodes petola (Blume) Lindl. & BMHEP-2315 \\
\hline 172 & Malleola sp. & AMHEP-0598 \\
\hline 173 & $\begin{array}{l}\text { Mycaranthes citrina } \\
\text { (Ridl.) Rauschert }\end{array}$ & $\begin{array}{l}\text { BMHEP-1616, CMHEP-2201, CMHEP-1330, } \\
\text { CMHEP-1344 }\end{array}$ \\
\hline 174 & Mycaranthes latifolia Blume & CMHEP-1327, CMHEP-1380 \\
\hline 175 & $\begin{array}{l}\text { Mycaranthes magnicallosa (Ames \& } \\
\text { C.Schweinf.) J.J.Wood }\end{array}$ & CMHEP-1342, CMHEP-0230 (endemic to Borneo) \\
\hline
\end{tabular}




\begin{tabular}{|c|c|c|}
\hline 176 & Mycaranthes obliqua Lindl. & $\begin{array}{l}\text { CMHEP-1478, CMHEP-1334, CMHEP-0650, } \\
\text { BMHEP-4054, AMHEP-889, BMHEP-04794 }\end{array}$ \\
\hline 177 & $\begin{array}{l}\text { Mycaranthes oblitterata } \\
\text { Blume }\end{array}$ & $\begin{array}{l}\text { CMHEP-0235, BMHEP-1682-1, BMHEP-1610, } \\
\text { CMHEP-2723, BMHEP-3859, BMHEP-3922, } \\
\text { BMHEP-04514, BMHEP-04942, CMHEP-05005, } \\
\text { CMHEP-5906, BMHEP-05704 }\end{array}$ \\
\hline 178 & $\begin{array}{l}\text { Mycaranthes pannea (Lindl.) S.C.Chen \& } \\
\text { J.J.Wood }\end{array}$ & CMHEP-1821, CMHEP-1353, СMHEP-05184 \\
\hline 179 & Mycaranthes sp. & CMHEP-2214, BMHEP-06395 \\
\hline 180 & Nephelaphyllum pulchrum Blume & BMHEP-04708 \\
\hline 181 & Neuwiedia borneensis de Vogel & CMHEP-06339 (endemic to Borneo) \\
\hline 182 & Neuwiedia veratrifolia Blume & CMHEP-6012 \\
\hline 183 & Oberonia cf. rubra Ridl. & AMHEP-0596 \\
\hline 184 & Oberonia ciliolata Hook f. & BMHEP-4017 \\
\hline 185 & $\begin{array}{l}\text { Oberonia insectifera } \\
\text { Hook. f. }\end{array}$ & AMHEP-1140 \\
\hline 186 & Oberonia padangensis Schltr. & $\begin{array}{l}\text { BMHEP-2229, BMHEP-2253, CMHEP-2257, } \\
\text { CMHEP-1377, AMHEP-884, CMHEP-05182 }\end{array}$ \\
\hline 187 & Oberonia spp. & BMHEP-4058, СMHEP-05176, СМHEP-6029 \\
\hline 188 & $\begin{array}{l}\text { Ornithochilus difformis (Wall. ex Lindl.) } \\
\text { Schltr. }\end{array}$ & BMHEP-4057 \\
\hline 189 & $\begin{array}{l}\text { Oxystophyllum sinuatum (Lindl.) } \\
\text { M.A.Clem. }\end{array}$ & AMHEP-1112 \\
\hline 190 & Oxystophyllum spp. & CMHEP-4296, CMHEP-5981 \\
\hline 191 & Pennilabium struthio Carr & AMHEP-597 \\
\hline 192 & Peristylus gracilis Blume & AMHEP-0437 \\
\hline 193 & Peristylus hallieri J.J.Sm. & $\begin{array}{l}\text { CMHEP-0515, BMHEP-1688, CMHEP-2786, } \\
\text { BMHEP-4004 (endemic to Borneo) }\end{array}$ \\
\hline 194 & Phaius sp. & BMHEP-2858, ВMHEP-2875, СMHEР-1399 \\
\hline 195 & $\begin{array}{l}\text { Phalaenopsis cornu-cervi (Breda) Blume } \\
\text { \& Rchb.f. }\end{array}$ & AMHEP-1199, CMHEP-0663, CMHEP-6525 \\
\hline 196 & Phalaenopsis maculata Rchb.f. & CMHEP-2788, CMHEP-6104 \\
\hline 197 & $\begin{array}{l}\text { Pholidota carnea } \\
\text { (Blume) Lindl. }\end{array}$ & BMHEP-2323, ВMHEP-2432 \\
\hline 198 & $\begin{array}{l}\text { Pholidota gibbosa } \\
\text { (Blume) Lindl. ex de Vriese }\end{array}$ & $\begin{array}{l}\text { AMHEP-1152-1, CMHEP-0043, CMHEP-1346, } \\
\text { BMHEP-04512, BMHEP-04727, BMHEP-04901, } \\
\text { CMHEP-6507, CMHEP-6540, CMHEP-05149 }\end{array}$ \\
\hline 199 & Pholidota imbricata Lindl. & CMHEP-6549 \\
\hline 200 & Pholidota sulcata J.J.Sm. & AMHEP-878, CMHEP-5917 \\
\hline 201 & Pholidota sp. & BMHEP-4122 \\
\hline
\end{tabular}




\begin{tabular}{|c|c|c|}
\hline 202 & $\begin{array}{l}\text { Phreatia densiflora } \\
\text { (Blume) Lindl. }\end{array}$ & CMHEP-0627 \\
\hline 203 & Pinalia aff. saccifera (Hook.f.) Kuntze & CMHEP-05165, СMHEP-6041 \\
\hline 204 & $\begin{array}{l}\text { Pinalia cepifolia } \\
\text { (Ridl.) J.J.Wood }\end{array}$ & $\begin{array}{l}\text { CMHEP- } 2478 \\
\text { (live plant planted in garden at dam site) }\end{array}$ \\
\hline 205 & $\begin{array}{l}\text { Pinalia floribunda } \\
\text { (Lindl.) Kuntze }\end{array}$ & AMHEP-1228-1, BMHEP-04904, BMHEP-04790-1 \\
\hline 206 & $\begin{array}{l}\text { Pinalia latibracteata } \\
\text { (Ridl.) J.J.Wood }\end{array}$ & CMHEP-1338 \\
\hline 207 & $\begin{array}{l}\text { Pinalia tenuiflora } \\
\text { (Ridl.) J.J.Wood }\end{array}$ & CMHEP-2183, СMHEP-6513, CMHEP-05153 \\
\hline 208 & $\begin{array}{l}\text { Pinalia xanthocheila } \\
\text { (Ridl.) W.Suarez \& Cootes }\end{array}$ & CMHEP-4233 \\
\hline 209 & Plocoglottis hirta Ridl. & $\begin{array}{l}\text { CMHEP- } 6013 \text { (endemic to Borneo; live plant } \\
\text { planted in garden at dam site) }\end{array}$ \\
\hline 210 & Plocoglottis plicata (Roxb.) Ormerod & CMHEP-6526, CMHEP-6550, CMHEP-6568 \\
\hline 211 & Plocoglottis sp. & $\begin{array}{l}\text { BMHEP-1703, CMHEP-2905, BMHEP-2859, } \\
\text { BMHEP-4000, BMHEP-04543 }\end{array}$ \\
\hline 212 & Poaephyllum sp. & $\begin{array}{l}\text { BMHEP-5712 (live plant planted in garden at dam } \\
\text { site) }\end{array}$ \\
\hline 213 & Podochilus microphyllus Lindl. & CMHEP-0258 \\
\hline 214 & $\begin{array}{l}\text { Podochilus cf. schistanthera } \\
\text { Schltr. }\end{array}$ & BMHEP-3877 \\
\hline 215 & Podochilus sp.1 & BMHEP-1550 \\
\hline 216 & $\begin{array}{l}\text { Pteroceras fragrans } \\
\text { (Ridl.) Garay }\end{array}$ & $\begin{array}{l}\text { CMHEP-5951 } \\
\text { (endemic to Borneo) }\end{array}$ \\
\hline 217 & Pteroceras teres (Blume) Holttum & CMHEP-2940 \\
\hline 218 & $\begin{array}{l}\text { Renanthera breviflora (Rchb.f.) R.Rice \& } \\
\text { J.J.Wood }\end{array}$ & BMHEP-3802 (new record) \\
\hline 219 & Renanthera elongata (Blume) Lindl. & CMHEP-1329 \\
\hline 220 & $\begin{array}{l}\text { Robiquetia transversisaccata (Ames \& } \\
\text { C.Schweinf.) J.J.Wood }\end{array}$ & $\begin{array}{l}\text { CMHEP-5901 } \\
\text { (endemic to Borneo) }\end{array}$ \\
\hline 221 & Robiquetia sp. & CMHEP-2263 \\
\hline 222 & Saccolabiopsis viridiflora Aver. & BMHEP-3889, BMHEP-4035 (new record) \\
\hline 223 & Spathoglottis aurea Lindl. & CMHEP-2163, CMHEP-1388 \\
\hline 224 & Spathoglottis plicata Blume & $\begin{array}{l}\text { BMHEP-2436, AMHEP-0466, BMHEP-3897, } \\
\text { CMHEP-6022 }\end{array}$ \\
\hline 225 & Stichorkis cf. gibbosa (Finet) J.J.Wood & AMHEP-1064, AMHEP-0443 \\
\hline 226 & Taeniophyllum sp. & AMHEP-1247 \\
\hline 227 & Tainia obpandurata H.Turner & AMHEP-2358 (new record) \\
\hline
\end{tabular}




\begin{tabular}{|c|c|c|}
\hline 228 & $\begin{array}{l}\text { Tainia paucifolia } \\
\text { (Breda) J.J.Sm. }\end{array}$ & $\begin{array}{l}\text { CMHEP-2740, BMHEP-3828, BMHEP-03668, } \\
\text { BMHEP-05749 }\end{array}$ \\
\hline 229 & Tainia scapigera (Hook.f.) J.J.Sm. & CMHEP-6050 (endemic to Borneo) \\
\hline 230 & Tainia speciosa Blume & CMHEP-0030 \\
\hline 231 & Tainia sp. & BMHEP-2857 \\
\hline 232 & Thecopus secunda (Ridl.) Seidenf. & BMHEP-04530 \\
\hline 233 & Thelasis macrobulbon Ridl. & AMHEP-1180, CMHEP-0510, CMHEP-1319 \\
\hline 234 & Thelasis micrantha (Brongn.) J.J.Sm. & BMHEP-3890 \\
\hline 235 & Thelasis pygmaea (Griff.) Lindl. & CMHEP-1438 \\
\hline 236 & Thrixspermum centipeda Lour. & $\begin{array}{l}\text { AMHEP-833, CMHEP-2177, CMHEP-5907, } \\
\text { BMHEP-3351 }\end{array}$ \\
\hline 237 & $\begin{array}{l}\text { Thrixspermum lingiae P.O'Byrne \& } \\
\text { Gokusing }\end{array}$ & AMHEP-1944 (endemic to Borneo) \\
\hline 238 & Thrixspermum pulchrum Carr & BMHEP-3892 (new record) \\
\hline 239 & Thrixspermum raciborskii J.J.Sm. & $\begin{array}{l}\text { CMHEP-1491, CMHEP-2266, BMHEP-3882, } \\
\text { CMHEP-6001 }\end{array}$ \\
\hline 240 & Thrixspermum ridleyanum Schltr. & $\begin{array}{l}\text { AMHEP-1098, CMHEP-2910, AMHEP-3195, } \\
\text { CMHEP-5952 }\end{array}$ \\
\hline 241 & $\begin{array}{l}\text { Thrixspermum scopa (Rchb.f. ex Hook.f.) } \\
\text { Holttum }\end{array}$ & CMHEP-1324 \\
\hline 242 & Thrixspermum tenuicalcar Carr & BMHEP-3835 (new record) \\
\hline 243 & Thrixspermum spp. & $\begin{array}{l}\text { CMHEP-2751, CMHEP-2818, BMHEP-2251, } \\
\text { BMHEP-3019, CMHEP-1352, CMHEP-05186, } \\
\text { CMHEP-05183 }\end{array}$ \\
\hline 244 & Trichoglottis persicina P.O'Byrne & $\begin{array}{l}\text { AMHEP-2840, CMHEP-2256, CMHEP-5994 } \\
\text { (endemic to Borneo; new record) }\end{array}$ \\
\hline 245 & Trichoglottis aff. scaphigera Ridl. & $\begin{array}{l}\text { BMHEP-0299, CMHEP-2941, AMHEP-2841, } \\
\text { CMHEP-2258, BMHEP-3996 }\end{array}$ \\
\hline 246 & Trichoglottis smithii Carr & BMHEP-6909 \\
\hline 247 & Trichoglottis sp. & CMHEP-1336 \\
\hline 248 & Trichotosia ferox Blume & CMHEP-6074 \\
\hline 249 & Trichotosia gracilis (Hook.f.) Kraenzl. & $\begin{array}{l}\text { CMHEP-0498, CMHEP-0254, CMHEP-2680, } \\
\text { CMHEP-2174, CMHEP-2204, CMHEP-2221, } \\
\text { BMHEP-3985, CMHEP-6005, CMHEP-3327 }\end{array}$ \\
\hline 250 & Trichotosia microphylla Blume & AMHEP-2830, BMHEP-1373 \\
\hline 251 & Trichotosia teysmannii (Hook.f.) Kraenzl. & AMHEP-1176 \\
\hline 252 & $\begin{array}{l}\text { Trichotosia vestita (Wall. ex Lindl.) } \\
\text { Kraenzl. }\end{array}$ & $\begin{array}{l}\text { BMHEP-1546, CMHEP-2916, BMHEP-2155, } \\
\text { AMHEP-2834, CMHEP-2213, CMHEP-03639, } \\
\text { CMHEP-05103, CMHEP-06202 }\end{array}$ \\
\hline 253 & Trichotosia spp. & $\begin{array}{l}\text { AMHEP-1170, CMHEP-0240, BMHEP-2600, } \\
\text { CMHEP-2203 }\end{array}$ \\
\hline
\end{tabular}




\begin{tabular}{|c|l|l|}
\hline 254 & Vanilla aff. borneensis Rolfe & BMHEP-3119 \\
\hline 255 & Vanilla sumatrana J.J.Sm. & CMHEP-5950 (new record) \\
\hline 256 & Vrydagzynea sp.1 & CMHEP-5986 \\
\hline 257 & Zeuxine purpurascens Blume & $\begin{array}{l}\text { BMHEP-5759 (live plant planted in garden at dam } \\
\text { site) }\end{array}$ \\
\hline 258 & Unknown species (two species) & $\begin{array}{l}\text { BMHEP-1605, CMHEP-2634, CMHEP-05049, } \\
\text { BMHEP-05735, CMHEP-06608 }\end{array}$ \\
\hline
\end{tabular}

